OPEN ACCESS

Edited by:

Meng Zhou,

Wenzhou Medical University, China

Reviewed by:

Prashanth N. Suravajhala, Birla Institute of Scientific Research,

India

Yu-Long Wang,

Fudan University, China

*Correspondence: Shuping Zhao dczhshp8@126.com

Xianrang Song

basiclab@163.com

${ }^{\text {t}}$ These authors have contributed equally to this work

Specialty section: This article was submitted to

Cancer Genetics,

a section of the journal

Frontiers in Oncology

Received: 21 May 2020 Accepted: 22 September 2020 Published: 14 October 2020

Citation:

Zhang Z, Tang Y, Song X, Xie L, Zhao $S$ and Song $X$ (2020) Tumor-

Derived Exosomal miRNAs as Diagnostic Biomarkers in Non-Small

Cell Lung Cancer.

Front. Oncol. 10:560025.

doi: 10.3389/fonc.2020.560025

\section{Tumor-Derived Exosomal miRNAs as Diagnostic Biomarkers in Non-Small Cell Lung Cancer}

\author{
Zhijun Zhang ${ }^{1 \dagger}$, Youyong Tang $^{2 \dagger}$, Xingguo Song ${ }^{3}$, Li Xie $^{2}$, Shuping Zhao ${ }^{1 *}$ \\ and Xianrang Song ${ }^{2 *}$ \\ ${ }^{1}$ Department of Clinical Laboratory, Taian City Central Hospital, Taian, China, ${ }^{2}$ Department of Clinical Laboratory, Shandong \\ Cancer Hospital and Institute, Shandong First Medical University and Shandong Academy of Medical Sciences, Jinan, China, \\ ${ }^{3}$ Shandong Provincial Key Laboratory of Radiation Oncology, Shandong Cancer Hospital and Institute, Shandong First \\ Medical University and Shandong Academy of Medical Sciences, Jinan, China
}

Background: Delayed diagnosis is the main obstacle to improve prognosis of non-small cell lung cancer (NSCLC). Novel biomarkers for the diagnosis of NSCLC are urgently needed. This study aimed to identify the specific exosomal miRNAs with diagnostic and prognostic potential in NSCLC patients.

Materials and Methods: Transmission electron microscopy (TEM), qNano and western blots were used to characterize the exosomes isolated from the serum of NSCLC patients $(n=330)$ and healthy donors $(n=312)$ by ultracentrifugation. Exosomal miRNAs were profiled by miRNA microarrays and verified by quantitative PCR (qPCR). The diagnostic accuracy was determined by receiver operating characteristic $(\mathrm{ROC})$ analysis.

Results: A total of differential 22 miRNAs were screened out based on $\mathrm{P}<0.05$ and fold difference $>2.0$ by miRNA microarrays, among which, exosomal miR-5684 and miR-125b$5 p$ were significantly down-regulated in NSCLC patients compared to healthy donors, processing favorable diagnostic efficiency for (early) NSCLC. Importantly, the exosomal miR-125b-5p were associated with metastasis $(P<0.0001)$, chemotherapeutic effect $(P=0.007)$ and survival $(P=0.008)$.

Conclusion: Exosomal miR-5684 and miR-125b-5p levels are significantly downregulated in NSCLC patients, and serve as the promising diagnostic and prognostic biomarkers for NSCLC.

Keywords: non-small cell lung cancer, serum, exosomes, MiR-5684, MiR-125b-5p, biomarker, diagnosis

\section{INTRODUCTION}

Non-small cell lung cancer (NSCLC), the most common type of lung cancer which accounts for about $80 \%$ of all lung cancer, is the leading cause of cancer-related deaths worldwide (1). Its high mortality rate is not changed even with recent advances in cancer treatment, largely because approximately two-thirds of patients present with metastatic tumors at the time of diagnosis (2). 
Therefore, there is an urgent need for more reliable biomarkers for the early diagnosis of NSCLC and the monitoring of its progression and response to anti-cancer therapy.

MicroRNAs (miRNAs), the short (19-24 nt) non-coding RNAs, post-transcriptionally regulate gene expression by directly binding to the 3' untranslated regions (UTRs) of complementary messenger RNA targets $(3,4)$. Dysregulation of miRNAs expression plays critical roles in the development and progression of different cancers by affecting multiple cellular processes including cell proliferation, apoptosis, survival, invasion, metastasis, and chemotherapeutic resistance $(5,6)$. MiRNAs can be stably present in the blood called circulating miRNAs in the form of Ago2-miRNA protein complexes to protect against RNase degradation and multiple freeze-thaw cycles (7). Therefore, the differentially expressed circulating miRNAs between lung cancer patients and healthy humans can be used as novel biomarkers for diagnosis, serving the non-invasive biomarkers for NSCLC.

Furthermore, these miRNAs have also been identified in the exosome of plasma and serum in a remarkably stable form and are protected from endogenous RNase activity (7). Exosomes are small (30-150 nm diameter) membrane-bound vesicles that are released by all cell types into body fluids such as saliva, urine, plasma and malignant effusions (8). They can transfer functional proteins, mRNAs, miRNAs and other bioactive molecules to receipt cells, thus playing an important role in intercellular communication (9, 10). Numerous studies have shown that miRNA isolated from circulating exosomes in NSCLC patients mirrors the miRNA pattern expressed in NSCLC tissue (11). Besides, almost all cell types can secrete exosomes under normal or stressful conditions, and cancer cells in particular are known to secrete more exosomes than normal cells of the same organ type (12). All these empower them useful to develop highly sensitive diagnostic strategies for rapid and non-invasive monitoring of the pathological conditions of cancer patients (13) although only a few paper regard NSCLC. For example, it has been reported that exosomal miR-146a-5p, as well as miR-23b-3p, miR-10b-5p, and miR-21-5p act as prognostic biomarkers for NSCLC patients $(14,15)$.

In the current study, we aimed to investigate the diagnostic and prognostic role of exosomal miRNAs in NSCLC. We screened out exosomal miR-5684 and miR-125b-5p using microarray and validated their expression in a large cohort, followed by the analysis for their diagnostic efficiency and predicting chemotherapy response assessment, thus providing the evidence of exosomal miR-5684 and miR-125b-5p as biomarkers for NSCLC.

\section{MATERIALS AND METHODS}

\section{Patients and Healthy Donors}

Total 330 NSCLC patients and 312 healthy donors between January 2019 and July 2019 at the Shandong Cancer Hospital and Institute were enrolled in this study. Written informed consent was obtained from all participants. Tumor staging was estimated according to the Eighth Edition AJCC Cancer Staging Manual (16). All patients didn't receive any anti-tumor treatment before peripheral blood collection, or suffer any other endocrine, immune, or metabolic diseases. The healthy donors did not present any disease. Patient characteristics are summarized in Tables $\mathbf{1}$ and $\mathbf{2}$. In addition, TCGA miRNA-Seq data of NSCLC patients (BCGSC miRNA Profiling) and the corresponding clinical data (TCGA, http://cance rgeno me.nih. gov) including age, gender, TNM stage, histopathology, survival etc. were downloaded.

\section{Isolation of Exosomes}

The exosomes were separated by ultracentrifugation as described previously (17). Briefly, the serum was centrifuged at 10,000 $\mathrm{g}$ at $4^{\circ} \mathrm{C}$ for $30 \mathrm{~min}$ to remove the cell debris, followed by ultracentrifugation at $100,000 \mathrm{~g}$ for $2 \mathrm{~h}$ at $4^{\circ} \mathrm{C}$ (Type $50.4 \mathrm{Ti}$ Rotor; Beckman Coulter) to collect exosomes. The exosome pellets were resuspended in PBS for further analysis.

TABLE 1 | Characteristics of NSCLC patients for differentially expressed serum exosomal miR-5684.

\begin{tabular}{llccc}
\hline Characteristics & & No. case & Median & P-value \\
\hline Age $(\mathrm{y})$ & $\leq 62$ & 178 & 5.0194 & 0.450 \\
Gender & $>62$ & 147 & 4.9296 & \\
& Male & 195 & 5.0727 & 0.051 \\
Smoking & Female & 130 & 4.8380 & \\
& Yes & 172 & 5.0590 & 0.150 \\
Drinking & No & 153 & 4.8886 & \\
& Yes & 101 & 4.4369 & 0.981 \\
Pathology diagnosis & No & 216 & 4.4401 & \\
& AC & 225 & 4.9976 & 0.983 \\
Lymph node metastasis & SCC & 101 & 4.9949 & \\
& Yes & 167 & 5.0468 & 0.203 \\
TNM staging & No & 142 & 4.8952 & \\
& I-II & 137 & 4.8539 & 0.072 \\
Distant metastasis & III-IV & 174 & 5.0731 & \\
& Yes & 101 & 4.9451 & 0.688 \\
& No & 210 & 4.9958 & \\
& & & &
\end{tabular}

AC, adenocarcinoma; SCC, squamous cell carcinoma.

TABLE 2 | Characteristics of NSCLC patients for differentially expressed serum exosomal miR-125b-5p.

\begin{tabular}{llccc}
\hline Characteristics & & No. case & Median & P-value \\
\hline Age $(\mathrm{y})$ & $\leq 62$ & 174 & 4.4270 & 0.839 \\
Gender & Male & 144 & 4.4526 & \\
& Female & 126 & 4.4422 & 0.944 \\
Smoking & Yes & 168 & 4.4690 & 0.607 \\
\multirow{2}{*}{ Drinking } & No & 150 & 4.4045 & \\
& Yes & 105 & 5.0184 & 0.659 \\
Pathology diagnosis & No & 219 & 4.9624 & \\
& AC & 220 & 4.3597 & 0.485 \\
Lymph node metastasis & SCC & 98 & 4.4527 & \\
& Yes & 159 & 4.6104 & $\mathbf{0 . 0 0 0 8}$ \\
TNM staging & No & 143 & 4.1816 & \\
& I-II & 136 & 4.1536 & $\mathbf{0 . 0 0 0}$ \\
Distant metastasis & III-IV & 168 & 4.6557 & \\
& Yes & 99 & 4.7659 & $\mathbf{0 . 0 0 0}$ \\
& No & 207 & 4.2471 &
\end{tabular}

AC, adenocarcinoma; SCC, squamous cell carcinoma.

Bold values: significant difference. 


\section{Transmission Electron Microscopy (TEM)}

The exosomes were placed on a copper grid with a $50 \mu \mathrm{l}$ drop of $1 \%$ glutaraldehyde, and transferred to $100 \mu$ distilled water after $5 \mathrm{~min}$. The grids were left undisturbed for $2 \mathrm{~min}$ and stained with $50 \mu$ oxalyl uranyl solution ( $\mathrm{pH} 7$ ) for $5 \mathrm{~min}$, and then placed on a glass dish covered with paraffin film on ice. The grids were rinsed seven times with distilled water for $2 \mathrm{~min}$ each and observed using a JEM-1200EX transmission electron microscope operating at $100 \mathrm{kV}$.

\section{qNano Assay}

The size and particle density of the exosomes were measured by TRPS (Tunable Resistive Pulse Sensing, Izon Science Ltd, Christchurch, New Zealand) according to the manufacturer's instructions. The density of the exosome preparation was normalized using $1 \times 10^{13}$ particles $/ \mathrm{ml}$ calibration beads (18). The data was analyzed using Izon Control Suite software v.3.3.2.2000 (Izon Control Suite version 3.3.2.2001; Izon Science).

\section{Western Blotting}

Twenty $\mu \mathrm{g}$ exosomal protein extracts were separated by $10 \%$ SDS-PAGE and transferred onto PVDF membranes (Millipore, Billerica, MA, USA). After blocking with 5\% skimmed milk for $2 \mathrm{~h}$, the membranes were incubated overnight with primary antibodies including anti-CD9, anti-GM130, anti-CD54 and anti-TSG101 (CST, Danvers, United State) at $4^{\circ} \mathrm{C}$ and then with the HRP-conjugated secondary antibody at room temperature for $1 \mathrm{~h}$. The protein bands were visualized using ECL blot detection reagent (P0018; Beyotime, Shanghai, China).

\section{Microarray Analysis}

Exosomes were obtained from $5 \mathrm{ml}$ peripheral blood from 1 healthy and 2 NSCLC patients and subjected to microarray. The samples were hybridized onto a miRCURYTM LNA array (v.19.0) after the exosomal RNAs were labeled by the miRCURY Hy3/Hy5 power labeling kit (Vedbaek, Denmark). The expression data were normalized using the miRNAs with intensities $\geq 30$. The significantly differential miRNAs were obtained using the criteria of fold change $\geq 2.0$. MiRNA expression levels between the samples were distinguished by hierarchical clustering.

\section{RNA Isolation and Quantitative Real-Time PCR (qRT-PCR)}

Exosomal RNA was extracted using the TRIzol reagent (Thermo Fisher Scientific, Carlsbad, USA), and reverse transcribed to cDNA using Mix-X miRNA first-strand synthesis kit (TaKaRa Bio, Nojihigashi, Kusatsu, Japan). qPCR was performed using TB-Green Premix Ex Taq II reagent (Takara Bio) on the LC480 (Roche Diagnostics, Germany). The relative gene expression levels were evaluated by the $\Delta \mathrm{Ct}$ method $\left(\mathrm{Ct}^{\text {miRNA }}-\mathrm{Ct}^{\mathrm{U} 6}\right)$ as previously described (19). U6 was used as an internal control (20). Each sample was analyzed in duplicates. The primers were as follows: Has-miR-5684: 5'-CCCTAGCCAGAGCAACA GAAA-3'; Has-miR-125b-5p: 5'-TCCCTGAGACCCTAA
CTTGTGA-3'; U6: 5'-TGGAACGCTTCACGAATTTGC-G -3', and 5'-GGAACGATACAGAGAAGATTAGC -3'.

\section{Statistical Analysis}

Statistical analysis was performed using GraphPad Prism 6.0 (GraphPad Software, San Diego, CA, USA) and SPSS 22.0 (IBM, Ehningen, Germany) software. The Kolmogorov-Smirnov test was carried out to check the normality of the distribution. The normally distributed numeric variables were evaluated by parametric test, while non-normally distributed variables were analyzed by Mann-Whitney test; One-way ANOVA test or Kruskal-Wallis $\mathrm{H}$ test was used in comparisons among more than two groups. Receiver operating characteristic (ROC) curves with the corresponding $\mathrm{C}$ statistics (area under the curve, AUC), based on the logistic models, were used to determine the corresponding cutoff points with the pathological diagnosis. Data were shown as median \pm SD. The overall survival (OS) of the high- and low-expression groups were compared by the Kaplan-Meier method and log-rank test. The univariable and multivariable Cox analysis was performed to test risk score. $\mathrm{P}<0.05$ was considered statistically significant, all tests were set as double-tailed.

\section{RESULTS}

\section{Identification of Isolated Serum Exosomes}

The serum exosomes isolated from NSCLC patients and healthy donors were characterized by TEM, qNano and western blots. As shown in Figures 1A, B, the exosomes had the typical oval shape and measured $50-150 \mathrm{~nm}$ in diameter, and the size range was homogenous. Consistently, the characteristic markers of exosomes including CD9, CD54 and TSG101 were enriched in the exosome but not in the whole cell extracts, whereas GM130 (the negative control) was not detected in the exosomal protein extract (Figure 1C).

\section{Exosomal miR-5684 and miR-125b-5p Are Markedly Downregulated in NSCLC}

The results of miRNA profiling for NSCLC have been reported previously (21), from which, we identified 22 differentially expressed miRNAs (DEMs) (8 downregulated and 14 upregulated) in the exosomes of NSCLC patients compared to that of healthy controls (Figure 2A). Gene Ontology (GO) and Kyoto Encyclopedia of Genes and Genomes (KEGG) analyses further predicted 30 signaling pathways that were enriched among these DEMs (Figures 2B, C), among which retinoic acid receptor binding and long-term potentiation were likely involved in the tumorigenesis and development of NSCLC.

Six of these 22 miRNAs were further validated by qRT-PCR in a cohort of 330 NSCLC patients (including 149 at the earlystage, Table S1) and 312 healthy donors. As shown in Figures 3A, B, miR-5684 and miR-125b-5p were significantly downregulated in the serum exosomes of the NSCLC patients compared to that of healthy donors $(P<0.0001$ for both), whereas miR-17-5p, miR-9-5p, miR-148-3p and miR-154-5p 
A

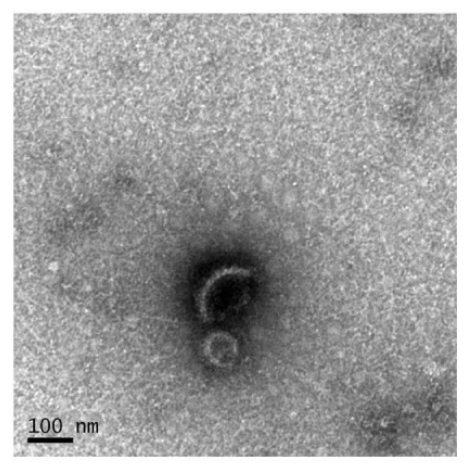

B

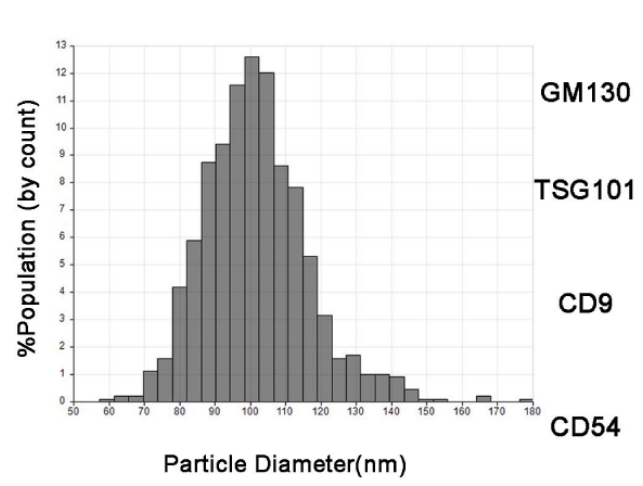

Cell Cell Exo Exo
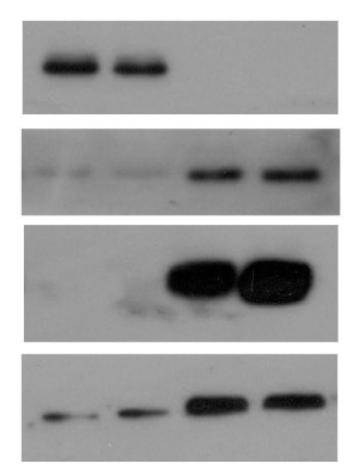

FIGURE 1 | Identification of isolated serum exosomes. (A) Representative TEM images of exosomes isolated from the sera of NSCLC patients. (B) Size distribution of exosomes ranging from 50-150 nm as analyzed by qNano system. (C) Immunoblots showing expression levels of the exosomal proteins CD9, CD54 and TSG101.

did not show any significant difference (Figure S1). Furthermore, exosomal miR-5684 and miR-125b-5p levels were markedly lower in the early-stage NSCLC patients compared to healthy donors $(P<0.0001$ and $P=0.002$ respectively; Figures 3C, D). However, they were irrelated with age, gender, smoking, drinking status and pathological features (Tables 1 and 2).

Notably, both miR-5684 and miR-125b-5p were stably upregulated in the exosomes compared to the exosomedepleted supernatant (EDS) $(\mathrm{P}=0.003$ and $\mathrm{P}=0.001$ respectively; Figure 4A), and unaffected by RNase A treatment (Figure 4B), or at room temperature for over 24 h (Figures 4C, D).

\section{Diagnostic Role of Serum Exosomal miRNAs in NSCLC Patients}

To evaluate diagnostic performance of exosomal miR-5684 and miR-125b-5p for NSCLC, a receiver-operating characteristic (ROC) curve was calculated (Tables S2 and S3). As shown in Figures 5A, B, the areas under the curve (AUCs) of exosomal miR-5864 and miR-125b-5p were 0.733 with sensitivity and specificity [95\% confidence interval (CI), 0.69-0.775], and 0.700 with sensitivity and specificity (95\% CI, 0.655-0.745) with $62.4 \%$ sensitivity and $70 \%$ specificity compared to the healthy donors. Meanwhile, the diagnostic performance of their combination was also calculated, possessing the AUC of 0.793 (95\% CI, $0.755-0.831$ ) with $82.7 \%$ sensitivity and $62.1 \%$, indicating exosomal miR-5684 and miR-125b-5p potentially act as the non-invasive circulating biomarkers for lung cancer (Figure 5C).

Similarly, when comparing the patients with early stage NSCLC to healthy controls, ROC curves demonstrated favorable diagnostic efficiencies of exosomal miR-5684 and miR-125b-5p, processing AUCs of 0.696 with $76.1 \%$ sensitivity and $54.9 \%$ specificity, 0.66 with $62.7 \%$ sensitivity and $63.6 \%$ specificity, respectively (Figures 5D, E). Moreover, the diagnostic performance for their combination demonstrated the AUC of 0.744 with a relative sensitivity of $80.6 \%$ and a relative specificity of $60.9 \%$ (Figure 5 F).

\section{The Combination of Serum Exosomal miRNA and Tumor Markers Increases Diagnostic Accuracy of NSCLC}

Carcinoembryonic antigen (CEA) is a pan-tumor prognostic marker and is recommended for diagnosing NSCLC (22). As shown in Figures 6A, B, miR-5684 or miR-125b-5p elevated the AUC of CEA significantly, from 0.791 to 0.85 or 0.839 . As expected, combining all three markers increased the AUC value to 0.877 (95\% CI, $0.848-0.906)$, and the sensitivity and specificity to 69.5 and $90.1 \%$ respectively (Figure 6C).

The cytokeratin 19 fragment CYFRA21-1 is an established circulatory tumor marker, and showed an AUC of 0.751. The combination of miR-5684 or miR-125b-5p and CYFRA21-1 had a higher AUC of 0.837 or 0.813 (Figures 6D, E). The combination of all three had a higher AUC of 0.863 (Figure 6F). Interestingly, combining either exosomal miR-5684 or miR125b-5p with CEA and CYFRA21-1 resulted in AUCs of 0.878 and 0.868 , respectively (Figures 6G, H). Importantly, the highest AUC of 0.896 (95\% CI, 0.869-0.923) was obtained with the combination of all of the above markers, and the sensitivity and specificity were correspondingly increased to 72.9 and $92.5 \%$ respectively (Figure 6I).

Likewise, the combination of CEA with serum exosomal miR$5684(\mathrm{AUC}=0.754)$ or miR-125b-5p $(\mathrm{AUC}=0.736)$ significantly improved the diagnostic efficiency of early-stage NSCLC compared to CEA alone (Figures 7A, B). The combination of all three further improved the diagnostic accuracy of early-stage NSCLC patients (AUC $=0.792(95 \%$ CI, $0.745-0.839 ; 75.4 \%$ sensitivity and $68 \%$ specificity) compared to healthy donors (Figure 7C). Similarly, the combination of miR-5684 or miR-125b-5 with CYFRA21-1 resulted in higher AUC (0.749 and 0.724, respectively) compared to CYFRA21-1 alone (Figures 7D, E). As expected, miR-5684 and miR-125b-5p 
A

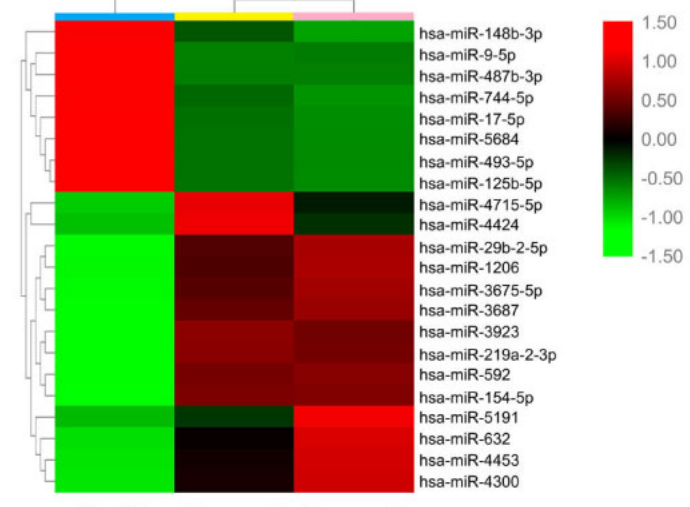

Heaith Cancer 1 Cancer 2

C
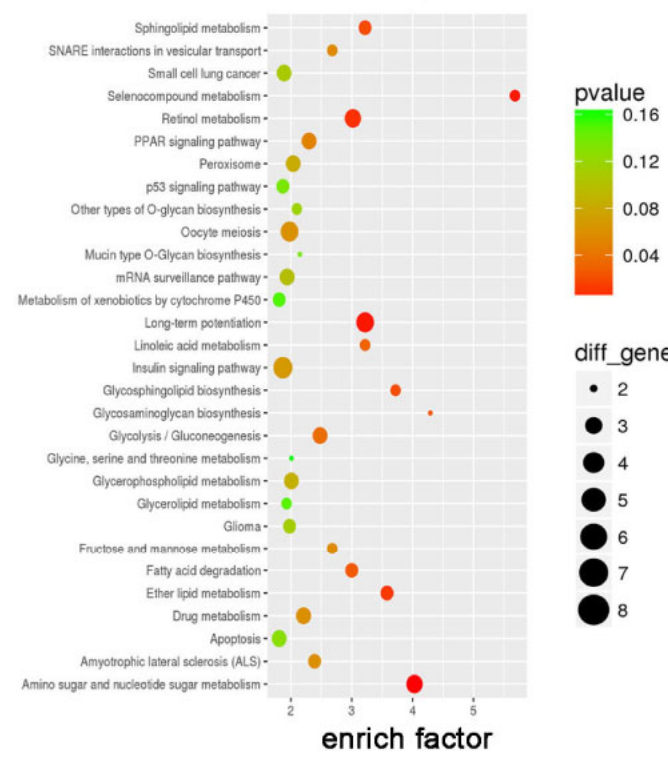

diff_gene_cou - 2

5

?
B

Top 30 of GO Enrichment

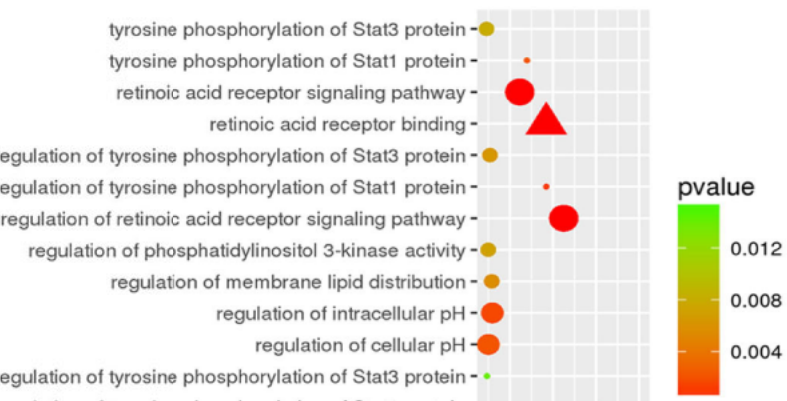
positive regulation of tyrosine phosphorylation of Stat1 protein positive regulation of tyrosine phosphorylation of STAT protein positive regulation of phosphatidylinositol 3 -kinase activity positive regulation of phosphatase activity positive regulation of lipid kinase activity phosphatidylinosito-4-phosphate binding $\mathrm{pH}$ reduction -
nucleotide-sugar metabolic process NLS-bearing protein import into nucleus negative regulation of retinoic acid receptor signaling pathway negative regulation of extrinsic apoptotic signaling pathway in absenc etc... metanephric nephron morphogenesis intracellular $\mathrm{pH}$ reduction disulfide oxidoreductase activity definitive hemopoiesis BH domain binding actin monomer binding -
GO_domain

- biological_process

- molecular_function diff_gene_coun - 4

- 6 enrich factor

FURE 2 I Serum exosomal miRNA profile of the NSCLC patients. (A) 
A

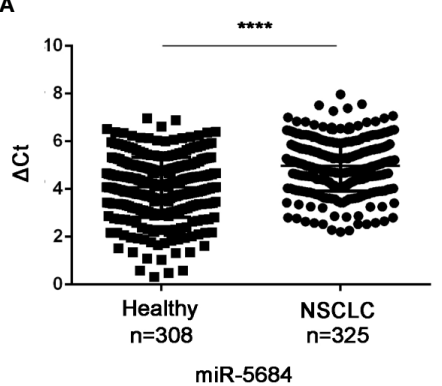

C

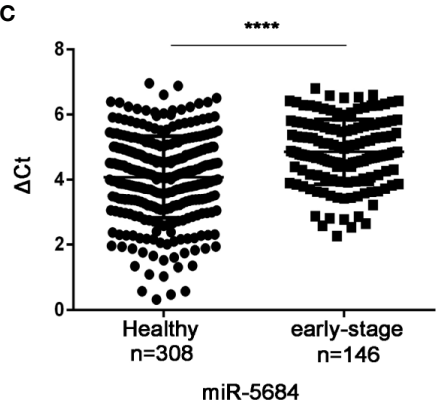

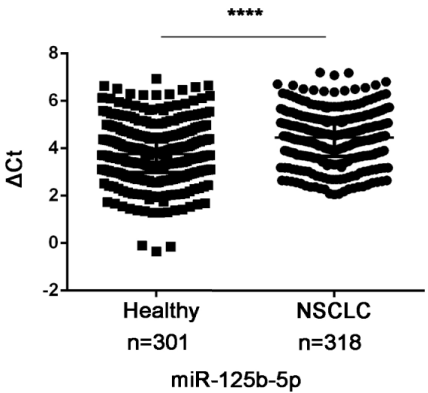

D

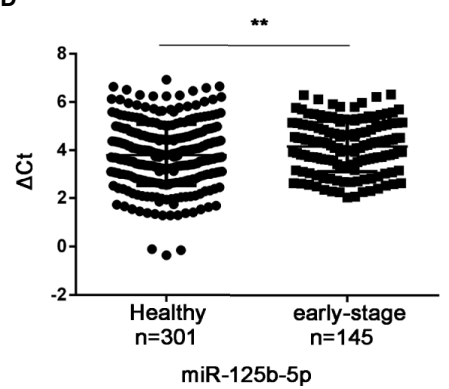

FIGURE 3 | Serum exosomal miR-5684 and miR-125b-5p are potential biomarkers of NSCLC. The expression levels of serum exosomal miR-5684 and miR-125b$5 p$ in (A) NSCLC patients and (B) healthy donors. The expression levels of serum exosomal miR-5684 and miR-125b-5p in (C) early-stage NSCLC patients and (D) healthy donors. $\left({ }^{\star \star \star *} \mathrm{P}<0.0001,{ }^{\star \star} \mathrm{P}<0.01\right)$.

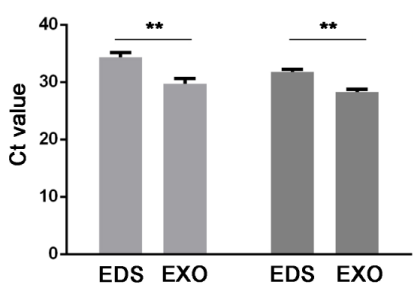

C

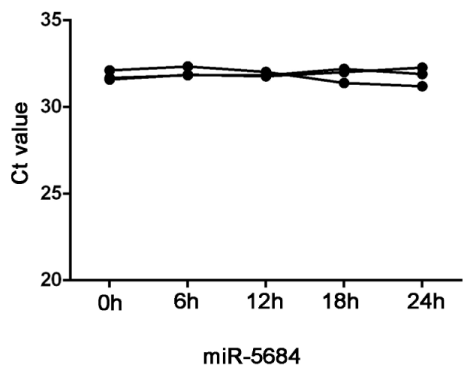

Incubation time at room tempreture
B

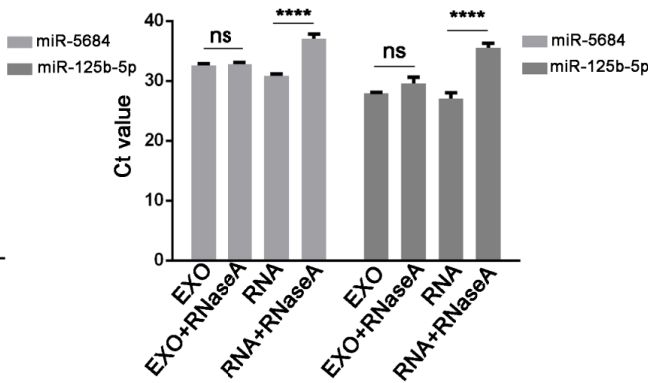

D

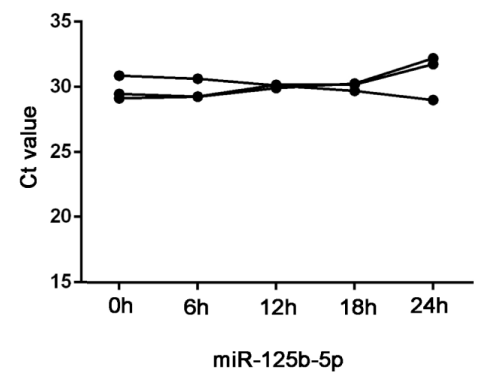

Incubation time at room tempreture

FIGURE 4 | Characteristics of serum exosomal miR-5684 and miR-125b-5p. (A) Expression levels of miR-5684 and miR-125b-5p from serum exosomes (EXO) and exosome-depleted supernatant (EDS). (B) Expression levels of both miRNAs in exosomes treated with RNase A or in isolated RNA. (C, D) Expression levels of the miRNAs in exosomes incubated at room temperature for different durations $\left({ }^{\star \star} \mathrm{P}<0.01,{ }^{\star \star \star \star} \mathrm{P}<0.0001\right.$, ns, not significant). 
A

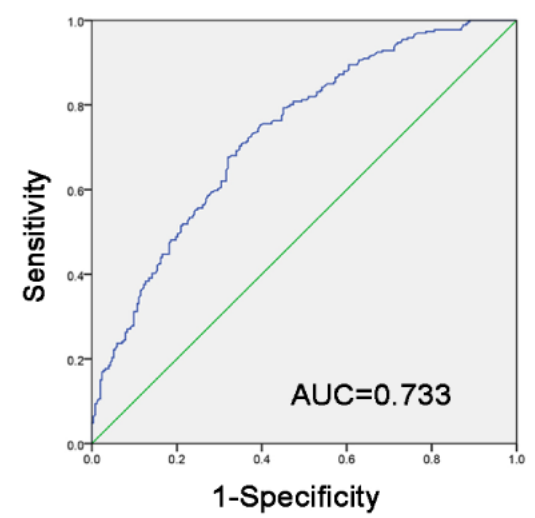

D

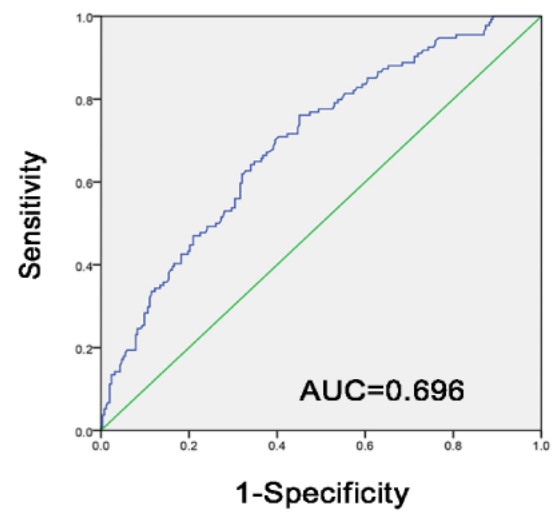

B

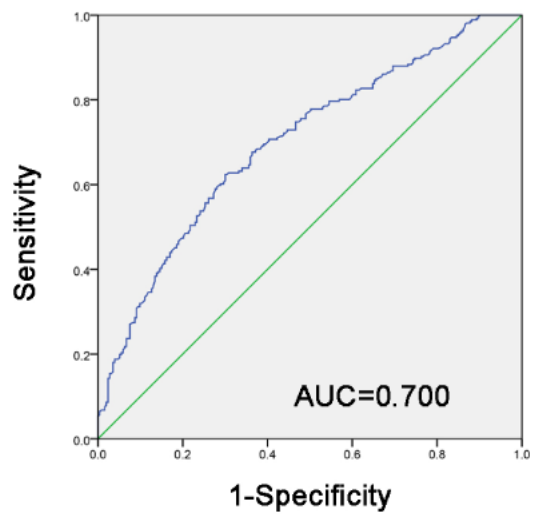

E

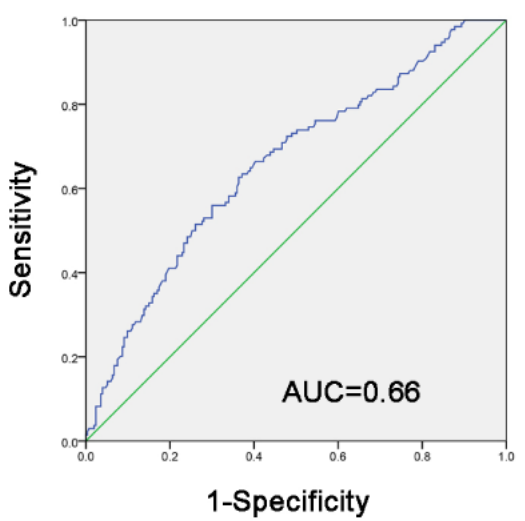

C

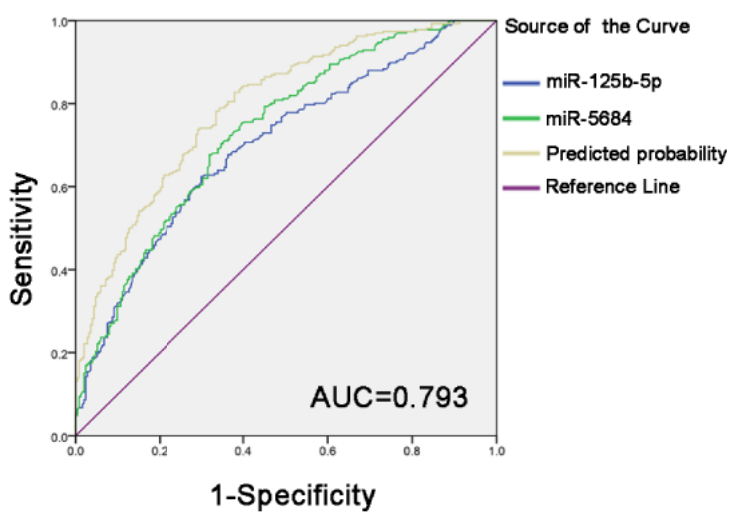

F

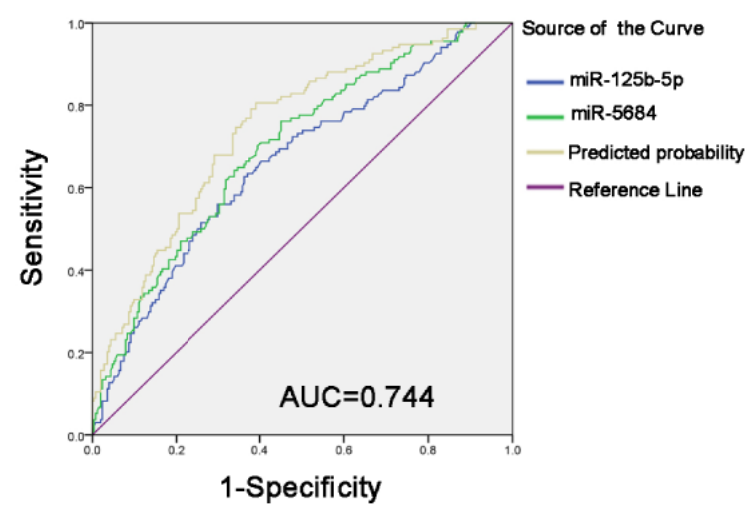

FIGURE 5 | Diagnostic role of serum exosomal miRNA expression levels in NSCLC patients. The AUCs of miR-5684 (A), miR-125b-5p (B), and both (C) in NSCLC patients relative to healthy donors. The AUCs of miR-5684 (D), miR-125b-5p (E), and both (F) in early-stage NSCLC patients relative to healthy donors. 
A

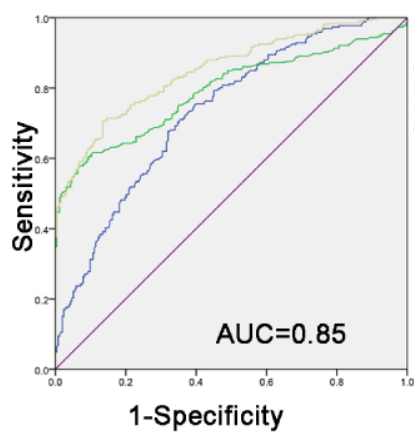

D

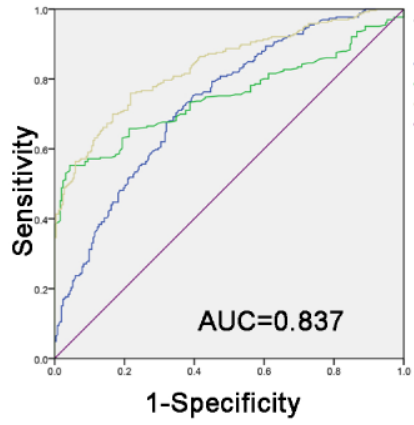

G

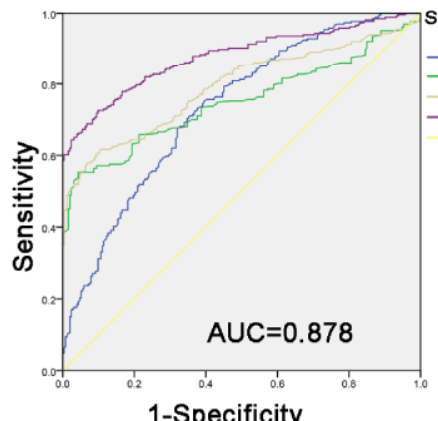

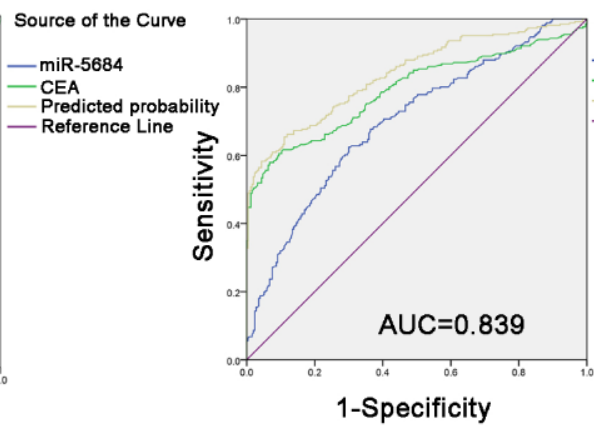

$\mathbf{E}$

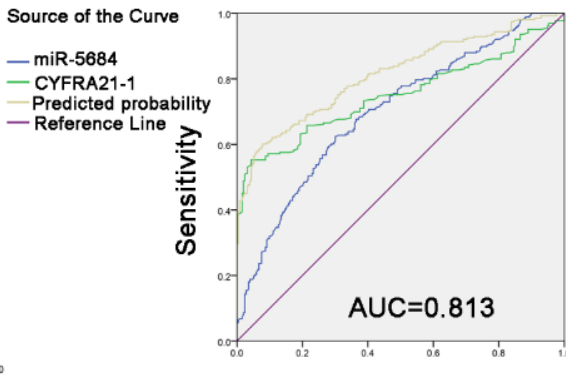

1-Specificity
C

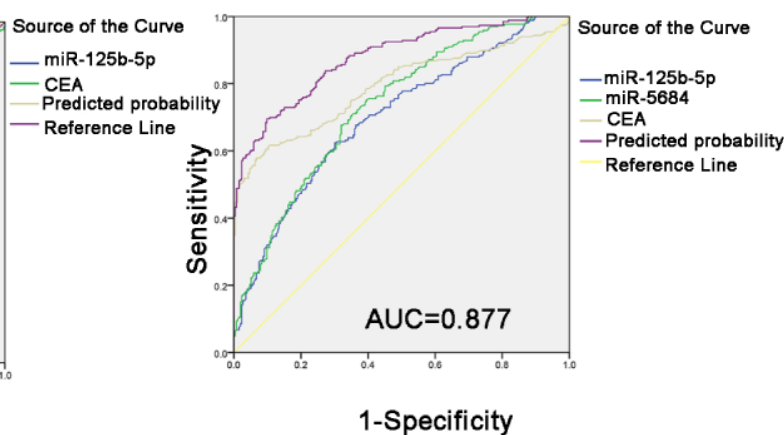

$\mathbf{F}$

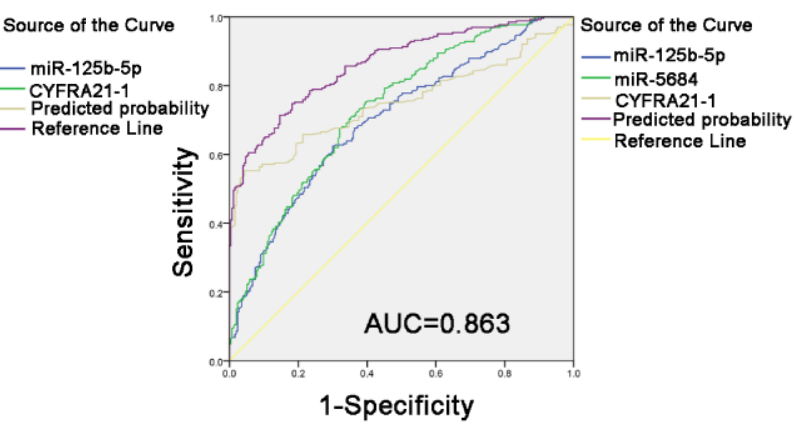

H

I

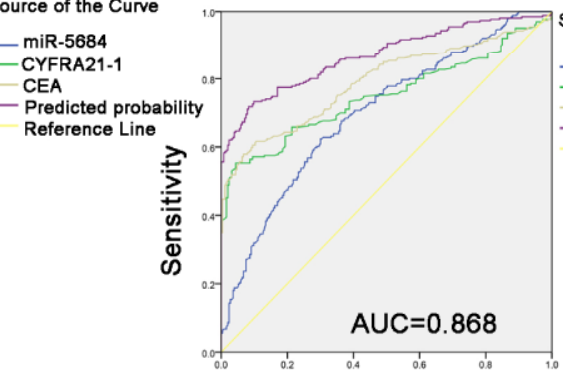

1-Specificity

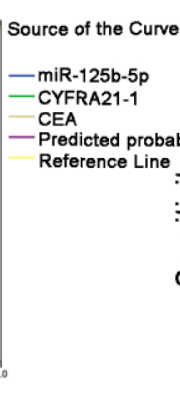

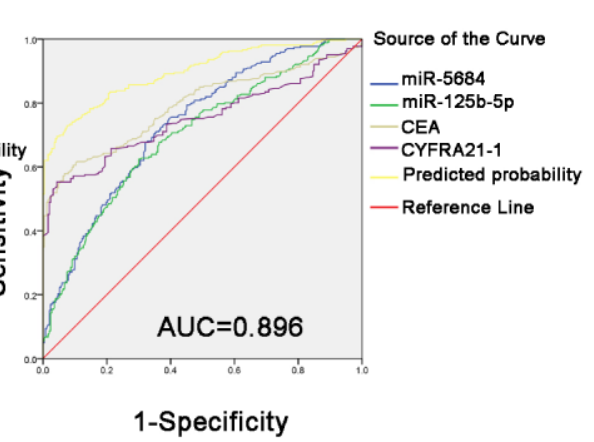

FIGURE 6 | Improved diagnostic capacity of serum exosomal miRNAs combined with established tumor markers in NSCLC patients. The AUCs of CEA combined with miR-5684 (A), miR-125b-5p (B), and both (C). The AUCs of CYFRA21-1 combined with miR-5684 (D), miR-125b-5p (E), and both (F). The AUCs of CEA and CYFRA21-1 combined with miR-5684 (G), miR-125b-5p (H), and both (I). 
A B

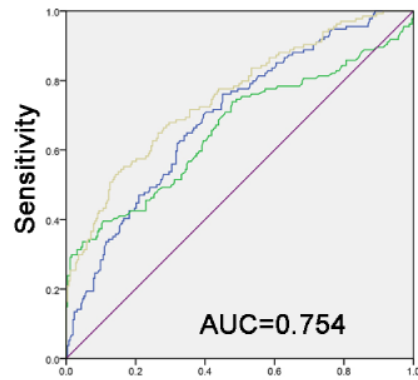

1-Specificity

D

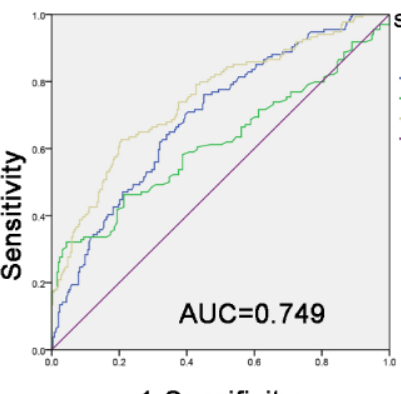

1-Specificity

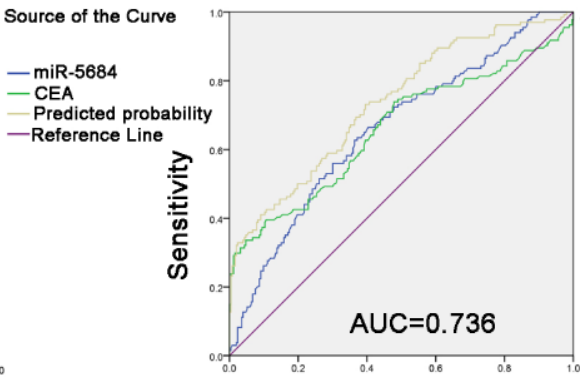

1-Specificity

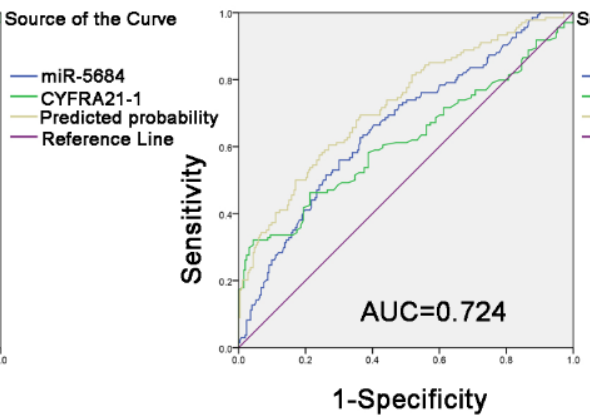

C
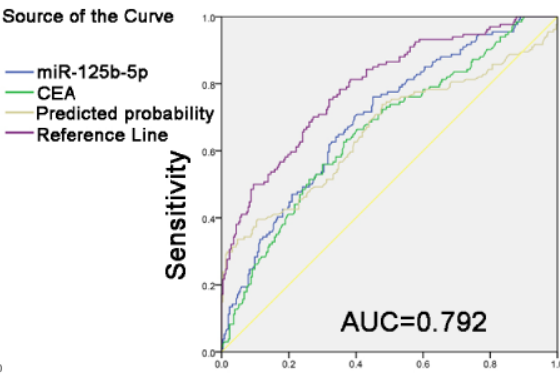

Source of the Curve

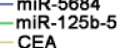

Predicted probability

Reference Line

1-Specificity

$\mathbf{F}$

。
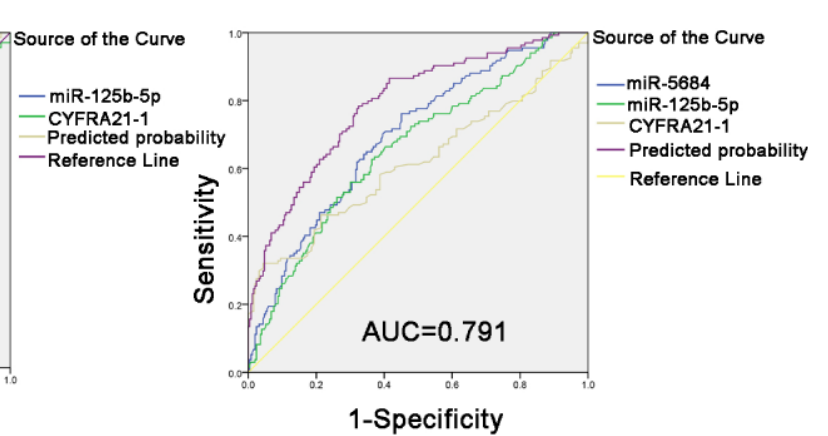

H

I

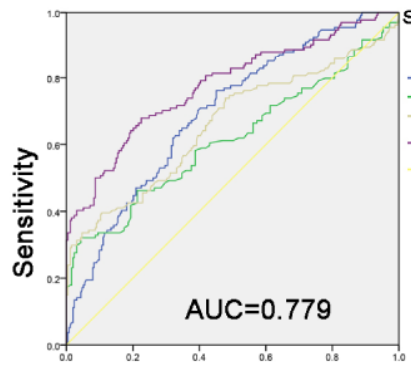

1-Specificity

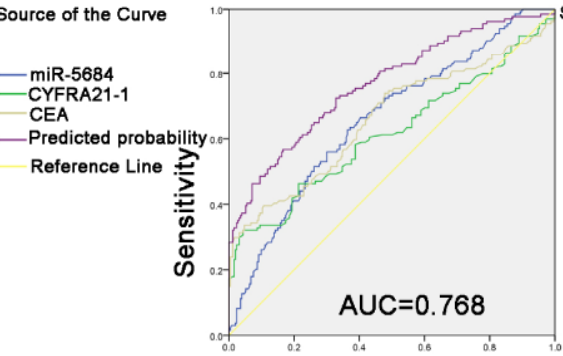

1-Specificity

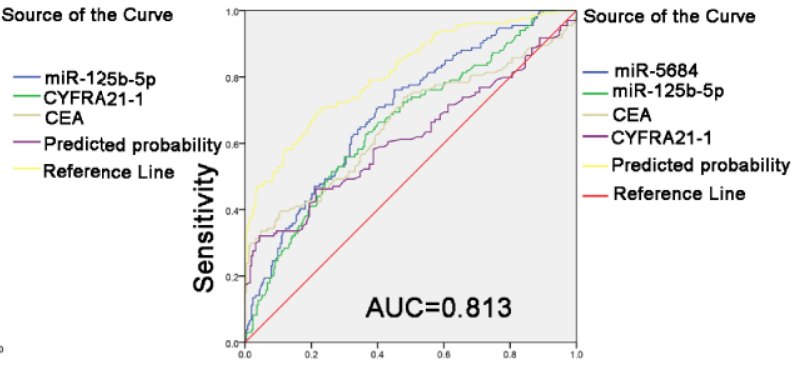

1-Specificity

FIGURE 7 | Improved diagnostic capacity of serum exosomal miRNAs combined with established tumor markers in early-stage NSCLC patients. The AUCs of CEA combined with miR-5684 (A), miR-125b-5p (B), and both (C). The AUCs of CYFRA21-1 combined with miR-5684 (D), miR-125b-5p (E), and both (F). The AUCs of CEA and CYFRA21-1 combined with miR-5684 (G), miR-125b-5p (H), and both (I). 
combined with CYFRA21-1 had a higher AUC of 0.791 for early stage NSCLC versus healthy subjects (Figure 7F). Furthermore, combination of miR-5684 or miR-125b-5p with CEA and CYFRA21-1 had an AUC of 0.779 and 0.768 (Figures 7G, H) respectively, whereas the AUC of all four markers was 0.813 (95\% CI, 0.767-0.858) with $69.4 \%$ sensitivity and $78.7 \%$ specificity (Figure 7I). Taken together, serum exosomal miR5684 and miR-125b-5p can increase the diagnostic efficacy of established markers like CEA and CYFRA21-1 for NSCLC as well as its early stages.

\section{Serum Exosomal miRNAs are Associated with the Tumor Stage}

Studies have shown that the plasma level of miR-125b-5p is related to the disease stage of the international staging system in multiple myeloma (23). In addition, miR-125b has been reported to be associated with the metastasis of liver cancer and breast cancer $(18,24,25)$. To determine the biological relevance of the serum exosomal miRNAs in NSCLC, we analyzed the relationship between their expression levels and the T/N stages. As shown in Figure 8A, exosomal miR-5684 expression was
A

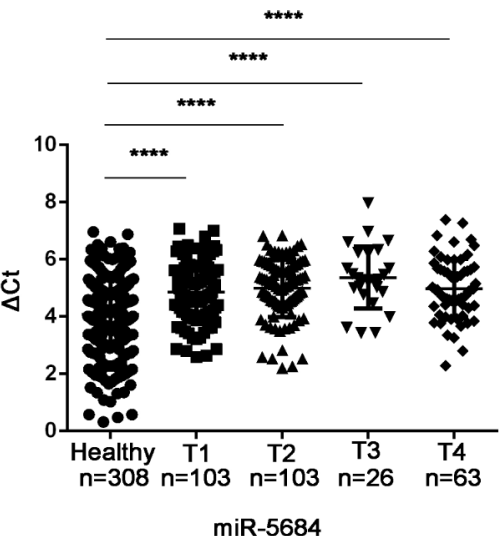

C

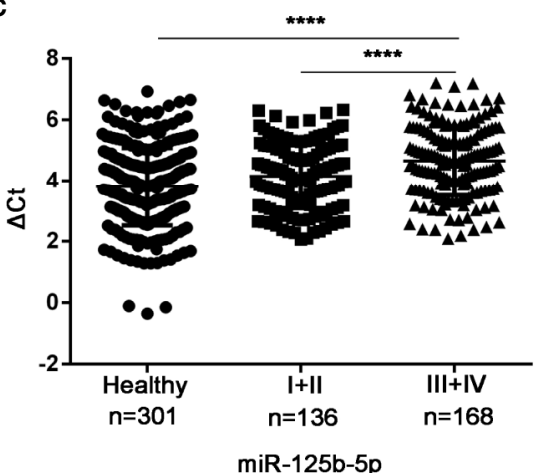

E

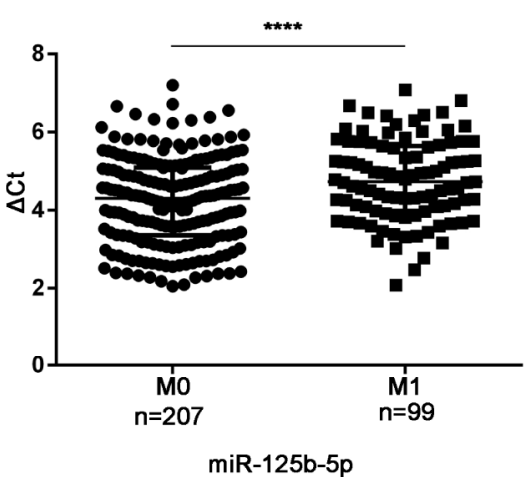

B
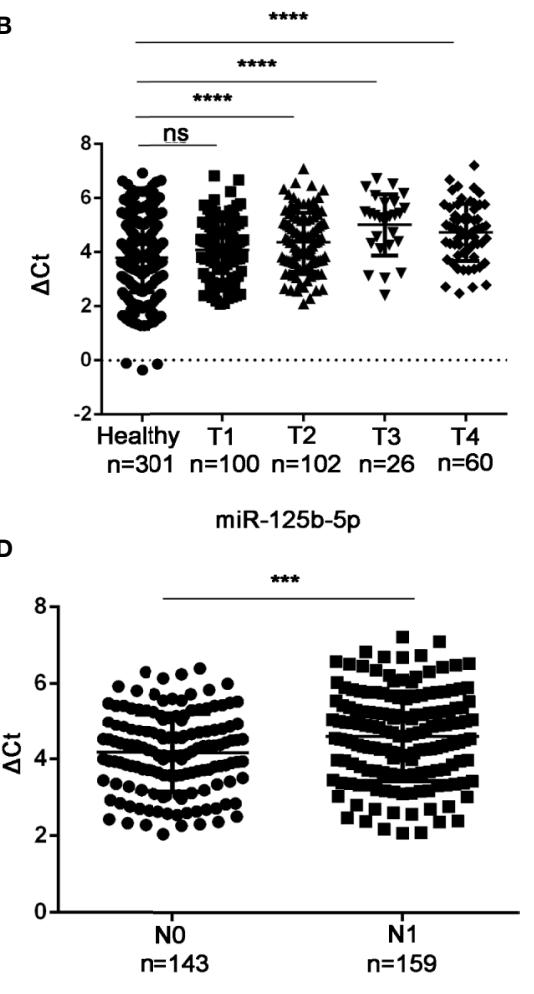

miR-125b-5p

$\mathbf{F}$

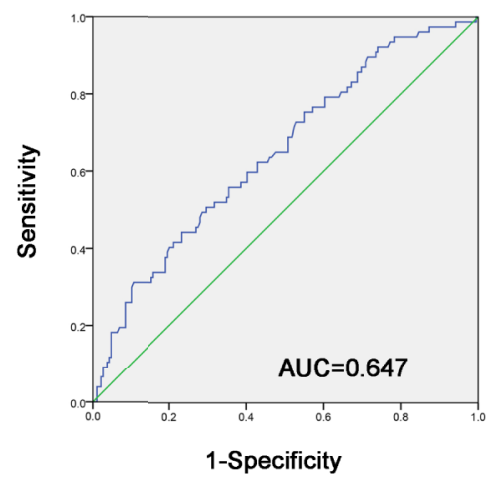

FIGURE 8 | Association between the serum exosomal miRNAs and tumor stage. Expression levels of (A) miR-5684 and (B) miR-125b-5p in T and N stages patients. (C) Serum exosomal miR-125b-5p expression in early stage (I+II) and advanced stage (III+IV) patients. (D, E) Statistical association between miR-125b-5p and (D) lymph node metastasis and (E) distant metastasis. (F) The AUC of serum exosomal miR-125b-5p was 0.647 in metastatic patients relative to non-metastatic NSCLC patients. ( ${ }^{\star \star \star} P<0.001,{ }^{\star \star \star \star} P<0.0001$, ns, not significant). 
significantly lower in the T1-T4 NSCLC patients compared to healthy honors, whereas that of exosomal miR-125b-5p was significantly lower in the patients at T2 and higher stages (Figure 8B). Furthermore, exosomal miR-125b-5p accurately discriminated the early stage (I+II) and advanced stage (III+IV) patients (Figure 8C), as well as the lymph node negative and lymph node positive groups (Figure 8D). In fact, high levels of exosomal miR-125b-5p was associated with significantly less lymphatic invasion $(P=0.0008)$ and distant metastasis $(P<$ 0.0001, Figure 8E). Finally, serum exosomal miR-125b-5p exhibited a high diagnostic accuracy for metastatic versus nonmetastatic patients with an AUC of 0.647 , and sensitivity and specificity $50.6 \%$ and $70.4 \%$ respectively. Taken together, serum exosomal miRNAs are useful biomarkers for early diagnosis of NSCLC and for predicting metastasis.

\section{Role of Exosomal miR-125b-5p in Chemotherapy Response Assessment}

Studies have shown that the expression level of miR-125b-5p correlates to the neoadjuvant chemotherapy response and prognosis of breast cancer $(26,27)$. In order to explore the relationship between miR-125b-5p and NSCLC chemotherapy response, 70 NSCLC patients who received first-line chemotherapy were added to our study. These patients' clinical response was graded according to the RECIST guidelines (28). Response rate (RR) is defined as the proportion of patients with complete remission (CR) and partial remission (PR) that can be evaluated as the best response; disease control rate (DCR) is defined as CR, PR and stable disease (SD) Proportion of patients with the best response (29). RR to the first-line chemotherapy in miR-125b$5 p$ was $42.9 \%$. In addition, DCR with the first-line chemotherapy in the exosomal miR-125b-5p was $82.9 \%$ (Table 3).

Furthermore, these patients were divided into PR and non-PR groups. Non-PR included SD and PD. The data showed that exosomal miR-125b-5p expression levels were lower in the non$\mathrm{PR}$ group than that in $\mathrm{PR}$ groups $(\mathrm{P}=0.007$, Figures 9A, B $)$. Thus, serum exosomal miR-125b-5p is a potential biomarker for predicting the chemotherapeutic response.

\section{Validating the Prognostic Value of Exosomal miR-125b-5p in NSCLC}

To further validate the prognostic relevance of miR-125b-5p in NSCLC, we analyzed the BCGSC miRNA profile data of 91 normal lung tissues and 991 NSCLC tissues from TCGA database. As shown in Figure 10A, miR-125b-5p expression was significantly decreased in the NSCLC tumors compared to the normal tissues $(\mathrm{P}=0.004)$. The patients $(\mathrm{n}=864)$ were then stratified into the miR-125b-5p high- or low-expression groups with median expression as the cut-off point. Patients expressing high levels of miR-125b-5p had significantly longer OS compared to the low-expression group ( $\mathrm{P}=0.008$; Figure 10B). Furthermore, univariate analysis showed that $\mathrm{T}$ stage $(\mathrm{P}=0.006)$, $\mathrm{N}$ stage $(\mathrm{P}=0.008), \mathrm{M}$ stage $(\mathrm{P}=0.001)$, TNM stage $(\mathrm{P}<0.0001)$ and miR-125b-5p expression levels $(\mathrm{P}=0.008)$ were significantly associated with the OS of NSCLC patients, whereas age $(\mathrm{P}=0.094)$, sex $(\mathrm{P}=0.371)$ and pathological status $(\mathrm{P}=0.501)$ had no significant influence. Multivariate analysis of the significant variables further indicated that only the miR-125b$5 \mathrm{p}$ expression level $[\mathrm{HR}=0.744,95 \%$ confidence interval $(\mathrm{CI})$ : $0.600-0.923 ; \mathrm{P}=0.007]$ was an independent prognostic factor for NSCLC (Table 4).

\section{DISCUSSION}

The poor prognosis of NSCLC patients is largely due to high recrudescence rates, reflecting the urgent needs of novel sensitive and specific biomarkers for early diagnosis. In current study, we screened out the exosomal miR-5684 and miR-125b-5p using microarray and validated their expression pattern in a large cohort, followed by the analysis for their diagnostic efficiency and predicting chemotherapy response assessment, thus providing

TABLE 3 | Response to the first chemotherapy in serum exosomal miR-125b-5p.

\begin{tabular}{lcccccc}
\hline Gene & N & CR & PR & SD & PD & RR \\
\hline miR-125b-5p & 70 & 0 & 30 & 28 & 12 & 42.9 \\
\hline
\end{tabular}

$C R$, complete response; $P R$, partial response; $S D$, stable disease; $P D$, progressive disease; $R R$, response rate; $D C R$, disease control rate.

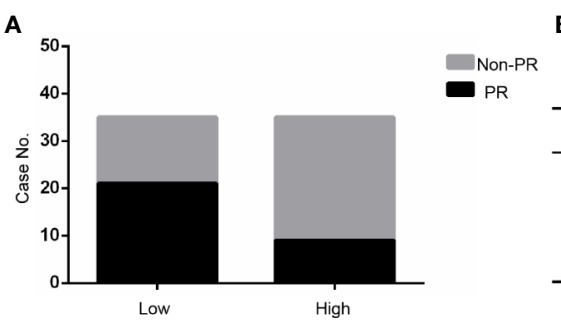

B

\begin{tabular}{cccccc}
\hline & & PR & non-PR & Total & P-value \\
\hline \multirow{3}{*}{ miR-125b-5p } & Low & 21 & 14 & 35 & \\
& High & 9 & 26 & 35 & 0.007 \\
& Total & 30 & 40 & 70 & \\
\hline
\end{tabular}

FIGURE 9 | The relationship between serum exosomal miR-125b-5p and chemotherapeutic effect. (A) High expression of serum exosomal miR-125b-5p was correlated with favorable first chemotherapy response. (B) Response to the 1ST treatment in patients. 

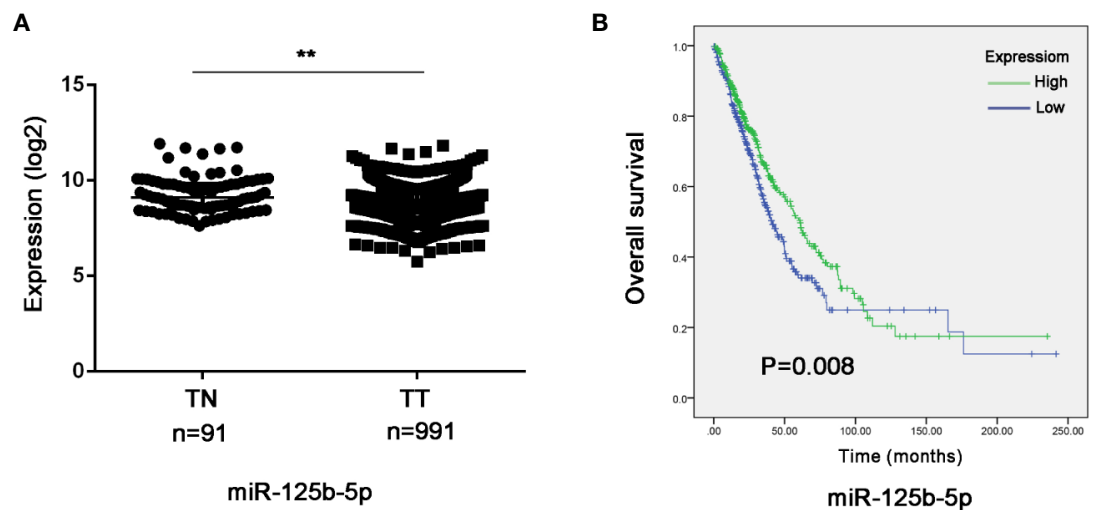

FIGURE 10 | Validation of the prognostic value of serum exosomal miR-125b-5p in NSCLC. (A) Relative expression of miR-125b-5p in NSCLC compared to norma tissue in TCGA database. (B) Kaplan-Meier survival analysis of TGCA patients with low-expression and high-expression miR-125b-5p groups. TN, normal tissue; TT, tumor tissue. ( $\left.{ }^{\star \star} \mathrm{P}<0.01\right)$.

the evidence of exosomal miR-5684 and miR-125b-5p as biomarkers for NSCLC.

MiR-125b has been reported previously in osteosarcoma (30), hematopoietic malignancies (31) and NSCLC (32). It is down-

TABLE 4 | The influence of different variables on OS for 864 patients with NSCLC analyzed by Cox proportional hazard model.

\begin{tabular}{|c|c|c|c|}
\hline \multirow[t]{3}{*}{ Variables } & \multicolumn{3}{|c|}{ OS } \\
\hline & \multirow{2}{*}{$\frac{\text { Univariate }}{\mathbf{P}}$} & \multicolumn{2}{|c|}{ Multivariate } \\
\hline & & HR (95CI\%) & $\mathbf{P}$ \\
\hline $\begin{array}{l}\text { Age } \\
\qquad 67 \\
\geq 67\end{array}$ & 0.094 & & Not included \\
\hline $\begin{array}{l}\text { Gender } \\
\text { Male } \\
\text { Female }\end{array}$ & 0.371 & & Not included \\
\hline T stage & 0.006 & & 0.925 \\
\hline T1 & & $0.778(0.127-4.771)$ & 0.786 \\
\hline T2 & & $0.823(0.135-5.002)$ & 0.833 \\
\hline T3 & & $0.971(0.158-5.950)$ & 0.974 \\
\hline $\mathrm{T} 4$ & & Reference & \\
\hline $\mathrm{N}$ stage & 0.008 & & 0.655 \\
\hline pNO & & Reference & \\
\hline $\mathrm{pN1}$ & & 0.835(0.309-2.259) & 0.655 \\
\hline M stage & 0.001 & & 0.829 \\
\hline $\mathrm{MO}$ & & $109.251(0-3.367 E+20)$ & 0.829 \\
\hline M1 & & Reference & \\
\hline Stage & $<0.0001$ & & 0.204 \\
\hline 1 & & $0.002(0-7.24 \mathrm{E}+15)$ & 0.780 \\
\hline$\|$ & & $0.003(0-9.351 E+15)$ & 0.789 \\
\hline III & & $0.005(0-1.488 E+16)$ & 0.806 \\
\hline IV & & Reference & \\
\hline $\begin{array}{l}\text { Pathology diagnosis } \\
\text { AC } \\
\text { SCC }\end{array}$ & 0.501 & & Not included \\
\hline $\operatorname{miR}-125 b-5 p$ & 0.008 & $0.744(0.600-0.923)$ & 0.007 \\
\hline
\end{tabular}

Bold values: significant difference. regulated in several malignancies and functions as a tumor suppressor in colorectal cancer, gallbladder cancer $(33,34)$, breast cancer (35) and esophageal squamous cell carcinoma (36), as well as suppresses bladder cancer development via inhibition of MALAT1 (32). Aberrant expression empowers miR-125b-5p the potential as the diagnostics and prognosis biomarkers. For instance, decreased serum levels of miR-125b$5 \mathrm{p}$ is associated with favorable prognosis in breast cancer (26, 27). MiR-125b-5p could serve as potential diagnostic biomarkers for ESCC and Multiple myeloma $(23,36)$. In current study, we demonstrated that these two exosomal miRNAs were downregulated in NSCLC group compared to healthy group with an AUC of 0.793 . Nevertheless, this is the first study to demonstrate a role of exosomal miR-5684 (https://www.genecards.org/cgibin/ carddisp.pl ?gene $=$ MIR5684\&keywords $=$ miR-5684) in cancer.

Notably, downregulation of serum exosomal miR-125b-5p in NSCLC patients also correlated to chemoresistance and disease progression, and patients that achieved a PR expressed significantly higher levels of this miRNA, indicating its role as a predictor for monitoring chemotherapy response. Our findings indicated high expression of miR-125b-5p was correlated with "favorable" 1st chemotherapy response, thus providing a noninvasive marker to predict 1st chemotherapy response. Besides, we also evaluated the prognostic value of miRNA125b-5p via analyzing the data from TCGA database. The higher expression of miR-125b-5p was associated with better OS, and lower level of miR-125b-5p level was associated with worse OS in lung cancer tissues. We assumed that exosomal miR-125b-5p could act as an independent predictor for NSCLC prognosis which needs further exploration.

The limitations of this study are the relatively small cohort size and lack of any mechanistic insights. Future studies ought to be conducted on larger cohorts to confirm our findings, and the mechanisms underlying the function of these exosomal miRNAs should also be elucidated through functional assays. In conclusion, serum exosomal miRNAs are promising non- 
invasive diagnostic and prognostic markers of NSCLC, and warrant clinical validation.

\section{DATA AVAILABILITY STATEMENT}

The raw data supporting the conclusions of this article will be made available by the authors, without undue reservation.

\section{ETHICS STATEMENT}

The studies involving human participants were reviewed and approved by the Shandong Cancer Hospital Affiliated to Shandong First Medical University and Shandong Academy of Medical Sciences of committee. All subjects gave written informed consent in accordance with the Declaration of Helsinki.

\section{AUTHOR CONTRIBUTIONS}

SZ and XrS designed the experiments. ZZ carried out the experiments. YT wrote the manuscript and prepared the

\section{REFERENCES}

1. Bray F, Ferlay J, Soerjomataram I, Siegel RL, Torre LA, Jemal A. Global cancer statistics 2018: GLOBOCAN estimates of incidence and mortality worldwide for 36 cancers in 185 countries. CA Cancer J Clin (2018) 68(6):394-424. doi: $10.3322 /$ caac. 21492

2. Nasim F, Sabath BF, Eapen GA. Lung Cancer. Med Clin North Am (2019) 103 (3):463-73. doi: 10.1016/j.mcna.2018.12.006

3. To KK. MicroRNA: a prognostic biomarker and a possible druggable target for circumventing multidrug resistance in cancer chemotherapy. J BioMed Sci (2013) 20:99. doi: 10.1186/1423-0127-20-99

4. Li J, Li X, Ren S, Chen X, Zhang Y, Zhou F, et al. miR-200c overexpression is associated with better efficacy of EGFR-TKIs in non-small cell lung cancer patients with EGFR wild-type. Oncotarget (2014) 5(17):7902-16. doi: 10.18632/oncotarget.2302

5. Di Leva G, Garofalo M, Croce CM. MicroRNAs in cancer. Annu Rev Pathol (2014) 9:287-314. doi: 10.1146/annurev-pathol-012513-104715

6. Zhang L, Li H, Yuan M, Li D, Sun C, Wang G. Serum Exosomal MicroRNAs as Potential Circulating Biomarkers for Endometriosis. Dis Markers (2020) 2020:2456340. doi: 10.1155/2020/2456340

7. Turchinovich A, Weiz L, Langheinz A, Burwinkel B. Characterization of extracellular circulating microRNA. Nucleic Acids Res (2011) 39(16):7223-33. doi: $10.1093 /$ nar/gkr254

8. Lasser C, Alikhani VS, Ekstrom K, Eldh M, Paredes PT, Bossios A, et al. Human saliva, plasma and breast milk exosomes contain RNA: uptake by macrophages. J Transl Med (2011) 9:9. doi: 10.1186/1479-5876-9-9

9. Colombo M, Raposo G, Thery C. Biogenesis, secretion, and intercellular interactions of exosomes and other extracellular vesicles. Annu Rev Cell Dev Biol (2014) 30:255-89. doi: 10.1146/annurev-cellbio-101512-122326

10. Li J, Liu K, Liu Y, Xu Y, Zhang F, Yang H, et al. Exosomes mediate the cell-tocell transmission of IFN-alpha-induced antiviral activity. Nat Immunol (2013) 14(8):793-803. doi: 10.1038/ni.2647

11. Reclusa P, Sirera R, Araujo A, Giallombardo M, Valentino A, Sorber L, et al. Exosomes genetic cargo in lung cancer: a truly Pandora's box. Transl Lung Cancer Res (2016) 5(5):483-91. doi: 10.21037/tlcr.2016.10.06 figures. XgS and LX contributed to analysis the experimental data. All authors contributed to the article and approved the submitted version.

\section{FUNDING}

This work was supported by the National Natural Science Foundation of China $(81773237,81672104,81972014)$, the Shandong Provincial Key Research and Development Program (2016GSF201146, 2017GSF18183 and 2017CXGC1207), Shandong Provincial Natural Science Foundation (ZR2019MH004 and ZR2019LZL016) and Science and technology development plans of Tai'an City (Project No. 2019NS213).

\section{SUPPLEMENTARY MATERIAL}

The Supplementary Material for this article can be found online at: https://www.frontiersin.org/articles/10.3389/fonc.2020. 560025/full\#supplementary-material

SUPPLEMENTARY FIGURE 1 | The expression levels of serum exosomal miRNAs in NSCLC patients and healthy donors. (A) miR-17-5p, (B) miR-9-5p, (C) miR-148-3p, and (D) miR-154-5p.
12. Hessvik NP, Llorente A. Current knowledge on exosome biogenesis and release. Cell Mol Life Sci (2018) 75(2):193-208. doi: 10.1007/s00018-017-2595-9

13. Nedaeinia R, Manian M, Jazayeri MH, Ranjbar M, Salehi R, Sharifi M, et al. Circulating exosomes and exosomal microRNAs as biomarkers in gastrointestinal cancer. Cancer Gene Ther (2017) 24(2):48-56. doi: 10.1038/cgt.2016.77

14. Yuwen D, Ma Y, Wang D, Gao J, Li X, Xue W, et al. Prognostic Role of Circulating Exosomal miR-425-3p for the Response of NSCLC to PlatinumBased Chemotherapy. Cancer Epidemiol Biomarkers Prev (2019) 28(1):16373. doi: 10.1158/1055-9965.EPI-18-0569

15. Liu Q, Yu Z, Yuan S, Xie W, Li C, Hu Z, et al. Circulating exosomal microRNAs as prognostic biomarkers for non-small-cell lung cancer. Oncotarget (2017) 8(8):13048-58. doi: 10.18632/oncotarget.14369

16. Amin MB, Greene FL, Edge SB, Compton CC, Gershenwald JE, Brookland RK, et al. The Eighth Edition AJCC Cancer Staging Manual: Continuing to build a bridge from a population-based to a more "personalized" approach to cancer staging. CA Cancer J Clin (2017) 67(2):93-9. doi: 10.3322/caac.21388

17. Wang N, Song X, Liu L, Niu L, Wang X, Song X, et al. Circulating exosomes contain protein biomarkers of metastatic non-small-cell lung cancer. Cancer Sci (2018) 109(5):1701-9. doi: 10.1111/cas.13581

18. Liang L, Wong CM, Ying Q, Fan DN, Huang S, Ding J, et al. MicroRNA-125b suppressesed human liver cancer cell proliferation and metastasis by directly targeting oncogene LIN28B2. Hepatology (2010) 52(5):1731-40. doi: 10.1002/ hep. 23904

19. Sudhakar Reddy P, Dhaware MG, Srinivas Reddy D, Pradeep Reddy B, Divya $\mathrm{K}$, Sharma KK, et al. Comprehensive evaluation of candidate reference genes for real-time quantitative PCR (RT-qPCR) data normalization in nutri-cereal finger millet [Eleusine Coracana (L.)]. PloS One (2018) 13(10):e0205668. doi: 10.1371/journal.pone.0205668

20. Shen X, Xue Y, Cong H, Wang X, Ju S. Dysregulation of serum microRNA574-3p and its clinical significance in hepatocellular carcinoma. Ann Clin Biochem (2018) 55(4):478-84. doi: 10.1177/0004563217741908

21. Wang N, Guo W, Song X, Liu L, Niu L, Song X, et al. Tumor-associated exosomal miRNA biomarkers to differentiate metastatic vs. nonmetastatic non-small cell lung cancer. Clin Chem Lab Med (2020) 58(9):1535-45. doi: 10.1515/cclm-2019-1329 
22. Ferrigno D, Buccheri G, Biggi A. Serum tumour markers in lung cancer: history, biology and clinical applications. Eur Respir J (1994) 7(1):186-97. doi: 10.1183/09031936.94.07010186

23. Jiang Y, Luan Y, Chang H, Chen G. The diagnostic and prognostic value of plasma microRNA-125b-5p in patients with multiple myeloma. Oncol Lett (2018) 16(3):4001-7. doi: 10.3892/ol.2018.9128

24. Zhou HC, Fang JH, Shang LR, Zhang ZJ, Sang Y, Xu L, et al. MicroRNAs miR$125 \mathrm{~b}$ and miR-100 suppress metastasis of hepatocellular carcinoma by disrupting the formation of vessels that encapsulate tumour clusters. J Pathol (2016) 240(4):450-60. doi: 10.1002/path.4804

25. Li Y, Wang Y, Fan H, Zhang Z, Li N. miR-125b-5p inhibits breast cancer cell proliferation, migration and invasion by targeting KIAA1522. Biochem Biophys Res Commun (2018) 504(1):277-82. doi: 10.1016/j.bbrc.2018.08.172

26. Liu B, Su F, Chen M, Li Y, Qi X, Xiao J, et al. Serum miR-21 and miR-125b as markers predicting neoadjuvant chemotherapy response and prognosis in stage II/III breast cancer. Hum Pathol (2017) 64:44-52. doi: 10.1016/ j.humpath.2017.03.016

27. Luo Y, Wang X, Niu W, Wang H, Wen Q, Fan S, et al. Elevated microRNA$125 \mathrm{~b}$ levels predict a worse prognosis in HER2-positive breast cancer patients. Oncol Lett (2017) 13(2):867-74. doi: 10.3892/ol.2016.5482

28. Schwartz LH, Litiere S, de Vries E, Ford R, Gwyther S, Mandrekar S, et al. RECIST 1.1-Update and clarification: From the RECIST committee. Eur J Cancer (2016) 62:132-7. doi: 10.1016/j.ejca.2016.03.081

29. Finn RS, Bengala C, Ibrahim N, Roche H, Sparano J, Strauss LC, et al. Dasatinib as a single agent in triple-negative breast cancer: results of an openlabel phase 2 study. Clin Cancer Res (2011) 17(21):6905-13. doi: 10.1158/ 1078-0432.CCR-11-0288

30. Liu LH, Li H, Li JP, Zhong H, Zhang HC, Chen J, et al. miR-125b suppresses the proliferation and migration of osteosarcoma cells through downregulation of STAT3. Biochem Biophys Res Commun (2011) 416(1-2):31-8. doi: 10.1016/j.bbrc.2011.10.117
31. Lin KY, Zhang XJ, Feng DD, Zhang H, Zeng CW, Han BW, et al. miR-125b, a target of CDX2, regulates cell differentiation through repression of the core binding factor in hematopoietic malignancies. J Biol Chem (2011) 286 (44):38253-63. doi: 10.1074/jbc.M111.269670

32. Han Y, Liu Y, Zhang H, Wang T, Diao R, Jiang Z, et al. Hsa-miR-125b suppresses bladder cancer development by down-regulating oncogene SIRT7 and oncogenic long non-coding RNA MALAT1. FEBS Lett (2013) 587 (23):3875-82. doi: 10.1016/j.febslet.2013.10.023

33. Sun M, Song H, Wang S, Zhang C, Zheng L, Chen F, et al. Integrated analysis identifies microRNA-195 as a suppressor of Hippo-YAP pathway in colorectal cancer. J Hematol Oncol (2017) 10(1):79. doi: 10.1186/s13045-017-0445-8

34. Yang D, Zhan M, Chen T, Chen W, Zhang Y, Xu S, et al. miR-125b-5p enhances chemotherapy sensitivity to cisplatin by down-regulating $\mathrm{Bcl} 2$ in gallbladder cancer. Sci Rep (2017) 7:43109. doi: 10.1038/srep43109

35. Ouyang M, Li Y, Ye S, Ma J, Lu L, Lv W, et al. MicroRNA profiling implies new markers of chemoresistance of triple-negative breast cancer. PloS One (2014) 9(5):e96228. doi: 10.1371/journal.pone.0096228

36. Mei LL, Wang WJ, Qiu YT, Xie XF, Bai J, Shi ZZ. miR-125b-5p functions as a tumor suppressor gene partially by regulating HMGA2 in esophageal squamous cell carcinoma. PloS One (2017) 12(10):e0185636. doi: 10.1371/journal.pone.0185636

Conflict of Interest: The authors declare that the research was conducted in the absence of any commercial or financial relationships that could be construed as a potential conflict of interest.

Copyright (c) 2020 Zhang, Tang, Song, Xie, Zhao and Song. This is an open-access article distributed under the terms of the Creative Commons Attribution License (CC BY). The use, distribution or reproduction in other forums is permitted, provided the original author(s) and the copyright owner(s) are credited and that the original publication in this journal is cited, in accordance with accepted academic practice. No use, distribution or reproduction is permitted which does not comply with these terms. 